\title{
Standardization of the Local/Regional Networks and Web Infrastructure for Database Processing, and Its Role in Development of the Regionally Integrated Businesses
}

\author{
Edmond Beqiri \\ University of Peja "Haxhi Zeka" \\ edmond.beqiri@unhz.eu \\ Genc Beqiri \\ PhD Candidate - University of Skopje \\ gencgb@evun.eu \\ Adem Tolaj \\ PhD Candidate - European University in Tirana
}

Doi:10.5901/ajis.2015.v4n2s1p98

\begin{abstract}
Most of the people who use web services and Internet network, are very little aware on computer network infrastructure, that enables to use the same information technology infrastructure in several countries of a region. With the evolution of new technology, networks have become more complex and they play a vital role in helping business performance. Taking advantage of technology, with the help of computer networks, new services can be added, and thereby will increase the productivity. This paper includes information on the importance of computer networks for the implementation and delivery of information based distributed computer networks. Developing applications for Internet / Intranet seeks to overcome the limitations of Web interface forms. Administrators of databases today, in Internet-based environment, should understand the implications of Web applications development for the database, in terms of: the type of extended support data, security of database, transaction management the database, and the design of the database. Internet/Intranet applications designed for larger companies, are complex because of of the segmented business widespread, they have more employees and they have the great variety of different functions.
\end{abstract}

Keywords: Internet, Intranet, data, management, transactions, security, database, heterogeneous networks.

\section{Introduction}

In recent times, in a few years, the Internet has become the global standard for communications. The Internet is a global network of computers that work in a complete TCP / IP protocol network. The web of the entire world or WWW (World Wide Web), is one of many services that works on the Internet; it brought the opportunity for access to documents with HTML format. Now, the Internet hosts millions of companies and Web sites of organizations that provide various kinds of services. Internet is marked as the new model in systems developers because it provides multiple benefits, such as universal access to information, common Graphical User interface, lower prices for the the preparation of final beneficiaries, and others. The Internet is a "free state system", where management of the base is delegated to the Governance System Data Base or Database Management System (GSDB or DBMS).

On the other side, the current IPv4 protocol became too complex since the various additional protocols must be installed with IPv4 for IP based equipments to operate properly. Address shortage is the most serious problem in IPv4. The number of Internet users in Europe and SEE countries is increasing. There are many new applications that require enormous number of IP addresses.

\section{Regional Networks and the Internet}

Internet is constructed to use virtually any type of environment transmitter, such as: Optical fiber lines, household equipment lines already in use, phone line, cable TV, as well other types of networks already prepared in advance, radio 
frequency and microwave channels, Wi-Fi technology, GPRS etc.

On the other hand, Internet can be accessed with almost any type of computer: Desktop computers, Laptop computers, HH Computer with WIFI network, HH Computer with GPRS network, Smart Cell Phones etc. Internet is also a platform that can be accessed with different operating systems: Windows Server, personal OS, Compact Os for the small memory device (for Small devices) etc.

Since the need for TCP / IP addresses has increased, the network has started to migrate from standard protocol IPv4 (Internet Protocol Version 4) with 8 bit value (32-bit addresses), to the new IPv6 protocol - more advanced than IPv4, the standard 12 bit value (128-bit address)

Some of the main reasons for the development of the new version of Internet protocol were spending an addressing space (with special emphasis on class B), rapid expansion of the backbone router tables Internet (Internet backbone routing Tables), security issues, limiting the options within the IPv4 protocol and the performance of the router. The most significant advantages of IPv6 to IPv4 protocol are:

IPv6 addressing model

Simplified Addressing equipment

Standard autoconfiguration (DHCPv6, Neighbor Discovery, etc.)

The fixed format of the head of package

Addressing Anycast

Improved Safety

The main advantage of IPv6 vs. IPv4 protocol is that the addresses are longer and create more space instead of nets. While an IP protocol IPv4 address consists of 32 bits and is treated as a homogenous unit, IPv6 protocol IP addresses consist of 128 bits (16 bytes), which allows for the inclusion of various types of services within the address. It is very evident that such an IP address is the unique address in 2128. The total number of addresses for IPv4 is $232=$ 4294967296, while the number for IPv6 is $2128=340.282 .366 .920 .938 .463 .463 .374 .607 .431 .768 .211 .456$. Comparisons between the size of an addressing space between these two protocols are the most varied:

If an addressing space IPv4 could be summed up in a handful of hand, the space equivalent to IPv6 would involve the entire volume of the planet.

space to 128 bits addressing is so great that every $\mathrm{mm} 2$ of land area can be entered from 155 billion IPv4 space.

The current IPv4 protocol became too complex since the various additional protocols must be installed with IPv4 for IP based equipments to operate properly. Address shortage is the most serious problem in IPv4. It is evident that there is a very big increase of number of Internet users in South East European countries. There are also new applications requiring enormous number of IP addresses.

IPv4 is designed based on the developments and requirements of the time and with the time some additional functions have been added in protocols, IPv6 has integrated into itself all added functions..

Figure 1: Network with IPv4 protocol stratification

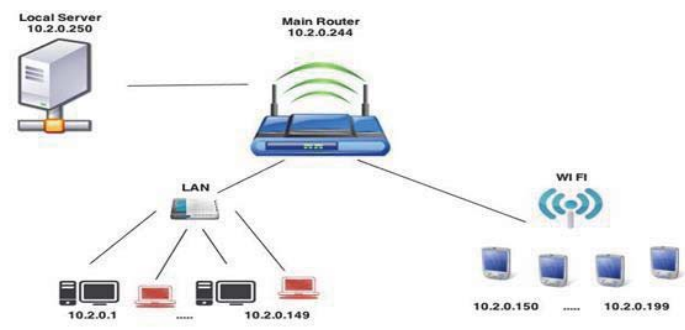

Source: Author contribution

In this paper we have took into account only the addresses needed for a particular job. Since we are dealing with different devices, this leads to the diversity of the databases used by these devices.

Databases are chosen to be SQL-based, but PC-S work with SQL database Server, and Handhelds work with compact version of SQL databases, specifically designed to occupy less possible memory (although it is compacting memory space and increase in size). 
This is essential for the equipment to perform with every document, and then to free memory for the future documents.

To achieve communication between handheld devices and databases to the local server, we use internet services. This communication is bidirectional.

\section{Internet Information Services}

\subsection{The interface of MS Windows Server operating system for processing from distance}

Once we provide a database on a server data processing from the Internet, everything depends on the environment provided for communication and processing support.

MS Windows Server operating systems provide various tools by which databases can be accessed.

One of the most useful tools is the Internet Information Services or IIS, which is very convenient because it comes as part of the Windows operating system, and therefore there is no need to supply add unique software.

Through Internet Information Services, it is possible to perform various jobs, which require connection to the Network. Internet Information Services software supports the development of World Wide WEB pages and it is in line with the Internet services such as:

FTP - File Transfer Protocol

World Wide Web interfaces

NNTP - Network News Transfer Protocol

SMTP - Simple Mail Transfer Protocol.

These functions enable communications between the units of an organization for the different requirements related to data transfer through the network.

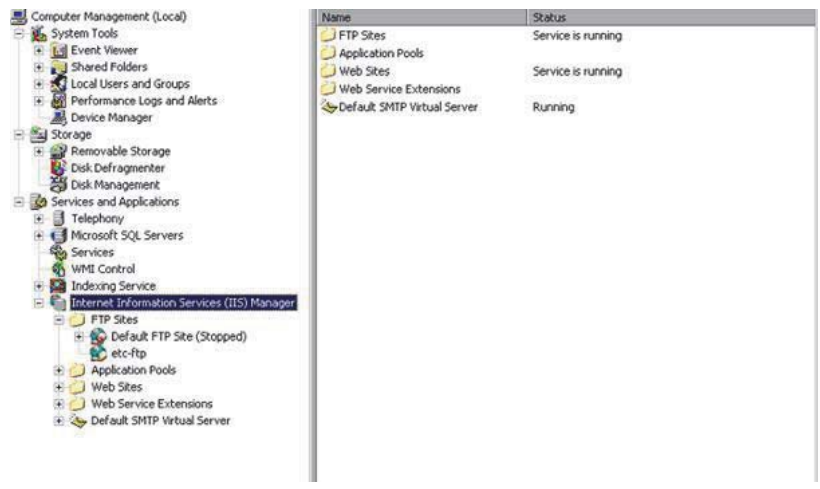

Figure 2: Appearance of Internet Information Services

Source: Author contribution

FTP - Unobstructed transfer of data

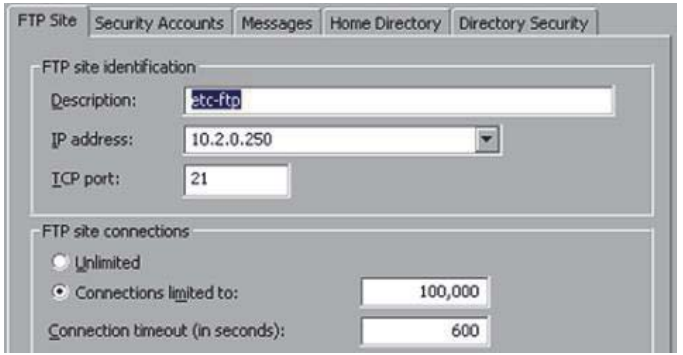

Figure 3: Internet Information Services

Source: Author contribution 
When we write the address of the Web site referred to, or symbolic address when we are active in the Internet / Intranet, we will gain access to data - database.

\subsection{Finding and refreshing of a commercial site}

All this happens under the procedures that are previously organized like as following:

The Browser requests for access to web pages,

Web Page is located in the Web Server,

Web server has scripts that allows connection with Database

Database Server provide connection to DBMS (SQL momentum)

SQL process the application

Results are returned to the Web Server

Web Server send the results to the Web Browser

Code that is used, is in Visual Basic Script - VBScript

$<\% @$ WebService Language="vb" Codebehind="MobSrv. asmx. vb" Class="MobSrv. iwMobSrv" \%>

With regard to the data server and database search, the program interface will issue processing stored procedures which would then turn their search results.

The advantage of this model used, is in the fact that everything is prepared for the local processing. At one point a part of its Application functionality is transferred to access the Internet. Of course we should follow special care to requests from the Internet which is "no state system".

\subsection{Web applications Server as a productive tool for the web based databases}

Web applications Server provides a consistent execution environment for the Web applications. Web applications server has multiple functions in the defined environment. Environment applications in the internet / intranet networks are used for:

Access and control the database from the Web site

Submit data from the database to the Web site, using a variety of used formats

Create Web sites for dynamic search

Create Web site to insert, update and delete records from database

Define required and optional relations

Define the forms required and optional fields

Enforce referential integrity fields format

Use simple and scalable queries and choose areas of application forms based on the business rules

\subsection{Corporate Network LAN organization within a country}

The Company that is located within a country has a network supplied by the same ISP. This has the advantage that the same rules apply to all units of the company, as per the technical aspects, as well as per organizational and legal aspects.

Organization of the enterprise depends on the physical distribution of the company and its units, business diversity, the number of employees as well as the technology used to prepare, apply and administer its business.

In the case of large companies this is crucial. 


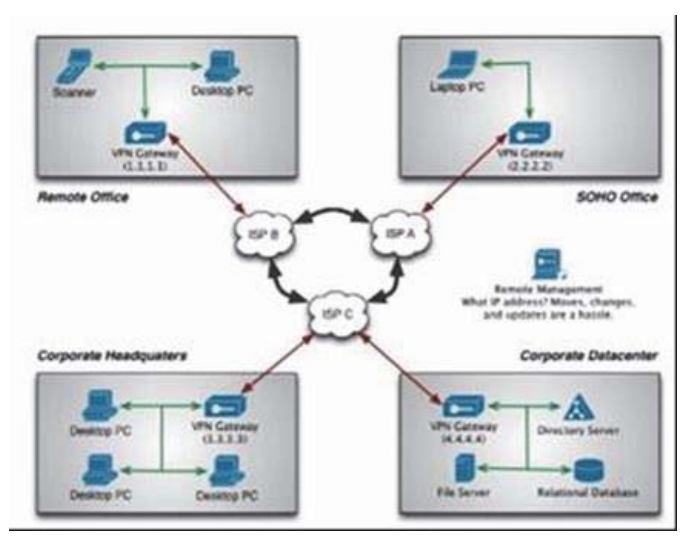

Figure 3: VPN Gateway iprovided with Static IP addresses

Source: Author contribution

The companies are dependent on services obtained from the providers, so their organization should be such that it reflects the way how to do the business.

This can be achieved through the organization with Virtual Private Networking (VPN). Virtual Private Networking tunnels, enables linking of two locations virtually, in a local private network. This enables the creation of our stratification within the Internet/Intranet network. The setting up of tunnels is directly based on the items among themselvesas with TCP/IP, as well as with the DNS infrastructure.

VPNs provides internal connection at all points of the enterprise, without the possibility of introducing risk exposure of the company from outside.

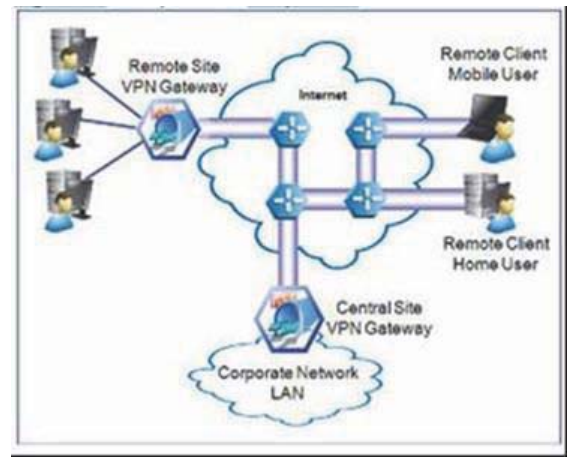

Figure 4: Sample of an Enterprise-Corporate network connected through the VPN servers

To access from outside within the company, we use the function NAT (Network Address Translation), that is the virtualization of Internet Protocol (IP) addresses, where a Public IP address through direct connection with an internal address allows controlled access from across the Internet network. This can be achieved through the authorization from the existing staff of the enterprise, also it has full control via the staff. All this procedure that is regulated to access from outside guarantees the security of transactions. 


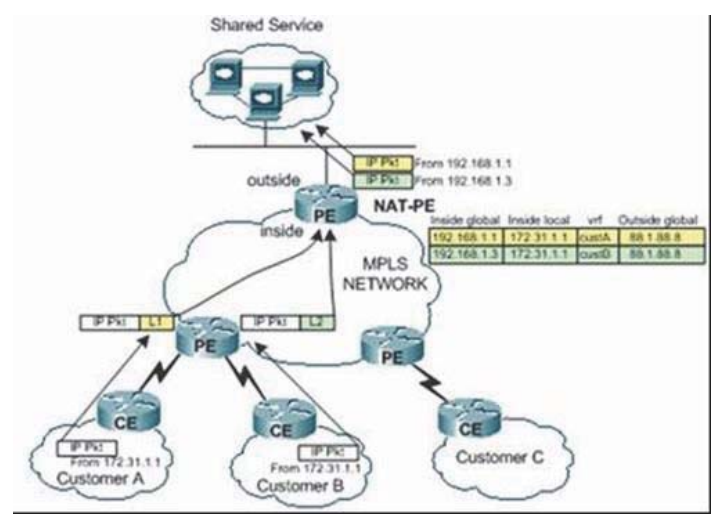

Figure 5: Network Address Translation (NAT) configured at the MPLS network PE router

Virtual Private Networking makes possible the use of:

Local Database Servers;

Local Web and Application Servers;

Data center servers from a distance;

Combination of Wired Networks with Wireless and GPRS Networks.

\section{Conclusion}

Regional enterprises which already have explored most of the traditional ways of providing services to customers, through new networked techniques can reach another form for the presentation and communication directly to the client.

This new enabled communication via Network technology, covers all kinds of communication between service providers with the existing and new customers.

Services for clients can be achieved in various ways:

Directly accessible via the Web clients

Customer Service with small shops (Customer Service)

Visits interaction with mobile devices (GPRS)

Services through system calls

Loyalty Card and smartcard model

etc.

The turnover that is reached, is always an impulse for the the further advancement of techniques and technologies used in intercommunications.

\section{References}

Adams, M. Mathis, "A System for Flexible Network Performance Measurement," INET, 2010

Abrams, B. NET Framework Standard Library Annotated Reference, Volume 2: Networking Library, Reflection Library, and XML Library, 2008.

Beaulieu, A. Learning SQL. O'Reilly Media Inc, Sebastopol CA, 2009.

Date, C.J. An Introduction to Database Systems, 8th Edition. Addison-Wesley, Reading MA, 2003.

Davidson, L. Ten Common Database Design Mistakes, http://www.simple-talk.com/sql/database-administration/ten-common-databasedesign-mistakes/, 2007.

Douglas E. Comer, "The Internet Book: Everything You Need to Know about Computer Networking and How the Internet Works", 2006, Prentice Hall College

Elmasri, R. and Navathe, S. Fundamentals of Database Systems, 6th Edition. Addison-Wesley, Reading MA, 2010.

Steffano Korper, Juanita Ellis; "The E-Commerce Book: Building the E-Empire"; 2007, Morgan Kaufmann 